\title{
PROYECTO DE INNOVACIÓN EDUCATIVA SOBRE LA FORMACIÓN PARA LAS PERSONAS QUE ACCEDEN A LA PRUEBA DE ACCESO A LA UNIVERSIDAD PARA MAYORES DE 25 AÑOS
}

Francisco Manuel Moreno-Lucas ${ }^{1}$ : Universidad Católica San Antonio de Murcia. España

fmoreno@pdi.ucam.edu

\section{RESUMEN}

La educación a lo largo de la vida es uno de los elementos claves del EEES, para la integración del concepto del aprendizaje permanente hacia la profesionalidad. Todos nacemos inacabados y es a base de las experiencias formales y personales, como nos vamos desarrollando como personas hasta la senectud. Es en el ámbito de la educación no formal, donde existe una mayor laguna a la hora de permitir el aprendizaje continuo de las personas adultas, por lo que la propuesta de un plan formativo innovador, que se presenta en materia de técnicas de aprendizajes en materia de estudio, adaptado a la características de los adultos, uno de los numerosos caminos de formación continua que lleva a la persona hacia la mejora del bienestar social, laboral y personal.

PALABRAS CLAVE: Educación no Formal - Estrategias de Aprendizaje - Educación Continua - Educación a lo largo de la Vida

\section{EDUCATIONAL INNOVATION PROJECT ON TRAINING FOR THOSE TEST ACCESS ACCESS TO COLLEGE FOR OVER 25 YEARS}

\begin{abstract}
Education throughout life is one of the key elements of the EHEA, the integration of the concept of lifelong learning towards professionalism. We are all born unfinished and based on personal experiences as formal as we develop our people to old age. In the

\footnotetext{
${ }^{1}$ Autor correspondiente

Francisco Manuel Moreno-Lucas: Profesor colaborador en la Universidad Católica San Antonio de Murcia. Murcia España

Correo: fmoreno@pdi.ucam.edu
} 
area of non-formal education, where there is a greater gap in allowing the continuous learning of adults, so that the proposed training plan in terms of innovative learning techniques in the field of specialized study to characteristics of adults, one of the many ways of training that leads people to improve social welfare, labor and personnel

KEY WORDS: Non-Formal Education - Continuing Education - Education throughout life - Learning Strategies.

\section{INTRODUCCIÓN}

La educación es un proceso que empieza con nuestro nacimiento y termina cuando dejamos esta vida, por lo que formal o informalmente estamos formándonos y educándonos a lo largo de toda nuestra existencia. Todos tenemos que aprender algo nuevo, a través del contacto con nuestro entorno, familia, amigos, comunidad, naturaleza, etc. El hombre como ser social necesita la interacción con los demás, y es en esa interacción, donde se da la formación, que la podemos concebir como la adquisición de conocimientos, habilidades, actitudes, a través del intercambio de experiencias.

La educación es importante y necesaria durante toda la vida, y ésta no se termina cuando finalizamos los estudios reglados, sino que uno se tiene que ir formando constantemente. Veo necesario, realizar una investigación en el campo de la formación continua en las personas adultas, que se plantea seguir estudiando, para dejar constatado lo importante que es estudiar y tener hábitos de estudios durante toda nuestra vida, tener presente que la formación continua es beneficioso para ti como para la sociedad, te dediques a la docencia u a otra ocupación.

Desde esta perspectiva existen diferentes opciones que para seguir formándonos a lo largo de nuestra vida, como la formación continua, la formación ocupacional y la formación en la empresa, siendo alternativas que nos ayudara a conseguir las metas propuestas, bajo una base de bienestar personal y social.

Para seguir adquiriendo conocimientos y una cultura actualizada sobre los continuos avances a nivel tecnológico, social y educativo, la formación continua es una opción muy atractiva para estar siempre activo

En esta línea de formación continua, el proyecto de innovación educativa de investigación, versara sobre la adquisición de técnicas y métodos de aprendizajes para las personas que acceden a la universidad mediante la prueba de acceso de mayores de 25 años.

Son muchas las dificultades que se encuentra este colectivo a la hora de volver a ponerse a estudiar, como el largo tiempo inactivo en el proceso de enseñanza/aprendizaje, poseer técnicas y hábitos de estudios obsoletos y poco efectivos, o simplemente tener dificultad para renovar y/o actualizar unas técnicas de 
aprendizajes olvidadas por el desuso. Estas personas, que deciden finalmente proseguir con sus estudios, necesitan una guía de estudio, un reciclaje en el ámbito educativo, unos métodos y técnicas de aprendizaje que le ayuden a adquirir nuevos hábitos y procedimientos educativos en su nueva vida como discentes.

En definitiva, estudiar no solo se refleja en el ámbito educativo, sino que se encuentre ya trabajando, o en casa, necesitas seguir ejercitando la memoria y "estudiando" todo lo nuevo que el día a día te brinda, por ello adquirir una buena base de estudio, le ayudara para la obtención más fácil de los nuevos avances sociales.

En nuestra sociedad se da bastante importancia a la educación permanente, una formación que nos acompaña durante toda nuestra vida, una educación que nos ayuda a adaptarnos a las nuevas tecnologías, a los cambios constantes de nuestra sociedad, una educación siempre encaminada a conseguir el bienestar personal y social del ser humano.

La aportación que se quiere realizar a través de este trabajo, es la elaboración de un documento explicativo, donde se refleje el porqué se debe de formar al colectivo que se presenta a las pruebas de acceso a la universidad para mayores de 25 años, en estrategias de aprendizaje y estudio, porque es necesario la formación continua en nuestra vida, dar a conocer las ventajas y los beneficios que nos puede dotar, tanto a nivel personal como a la sociedad, sobre todo al colectivo que lleva varios años sin actividad educativa.

Consideramos relevante, la producción de esta investigación para que se pueda conocer los diversos métodos de aprendizajes a desarrollar, el porqué es necesario dotar a las personas mayores de 25 años que quieren acceder a estudios universitarios, de técnicas, métodos, procedimientos, hábitos, en relación al estudio, que le ayuden tanto para superar la prueba de acceso a la universidad como para seguir estudiando los estudios superiores deseados.

Es conveniente, realizar previamente un recorrido teórico sobre la educación continua, como base de este proyecto, tener claro qué tipo de antecedentes existen, los ámbitos de educación donde se puede ubicar el futuro plan formativo, así como un esbozo sobre cuál será la estructura del proyecto innovador en educación que se presenta.

\subsection{Educación a lo largo de la vida}

La educación a lo largo de la vida fue una concepción que salió de una comisión que elaboro el informe Delors, donde se señala que en el siglo XXI, la educación tiene como un objetivo, el fomento de la formación en las personas desde la infancia hasta el final de su vida. Por lo tanto, se concibe ésta, como un tipo de formación que va más allá, del pensamiento anterior, de limitar la formación de la persona a un determinado intervalo de educación reglada.

Lo que se quiere con la educación a lo largo de la vida, tal y como menciona la 
UNESCO en 1996, es la de fomentar un tipo de educación, donde sea el propio hombre el agente de su formación, reestructurar el sistema educativo vigente en alas a una educación continua y duradera a lo largo de la vida, donde el hombre se vaya formándose a lo largo de su crecimiento, y no solo a raíz de su periodo escolar como se hacía en años anteriores.

Pero este tipo de educación lleva consigo numeroso cambios a nivel educativo, social, económico, etc., ya que debemos de adaptadnos a los cambios de nuestra sociedad, y con ello, crear nuevas formas de interacción donde las personas pueda seguir formándose

Esto requiere también de una nueva estructura educativa, donde se pueda desarrollar especialidades, especialistas, ámbitos de actuación, ya que no es lo mismo la formación de personas adultas en activo, como de las personas que están ya en su ciclo de senectud. La educación debe de estar presente, pero adaptada a cada situación.

Ya Platón, consideraba que la educación en el hombre debía prolongarse hasta la edad de cincuenta años, en la actualidad, los organismos internacionales preocupados por la educación, no solo lo corroboran, sino que lo prolonga hasta su expiración. Por ello, la educación a lo largo de la vida está ligada a la formación permanente, como una formación que comienza desde la edad infantil y que debe acompañar a la persona durante toda su vida, con el fin de ir alcanzando objetivos personales, sociales y profesionales.

Hoy en día la formación permanente es una realidad más que justificada, debido a los avances en todos los ámbitos (económicos, tecnológicos, sociales, políticos, etc.), es por ello necesario, la transformación de la educación con fines de dar respuesta a las necesidades que nos encontramos, de tal manera, la formación en el ámbito laboral es un hecho más que demostrable, donde la formación continua es imprescindible, por ejemplo, para que una empresa pueda seguir creciendo y ser competitiva. Pero también a nivel personal la educación continua tiene su relevancia, ya que los continuos avances tecnológicos en materia de bienestar social, te obligan a un reciclado con el propósito de no quedar obsoleto y disfrutar de las mejoras en la calidad de vida que nos ofrecen las nuevas tecnologías.

La formación a lo largo de la vida es ahora más que nunca necesaria, ya que estamos expuestos a continuos y rápidos cambios, que nos afectan tanto personal como laboralmente, y ante estas circunstancias, es más necesario la necesidad de formarse para comprenderlos y saber interpretarlo y con ello contribuir en el avance social.

En fin, el hombre es un ser no terminado, y de su formación dependerá, en mayor o menor medida, su futuro bienestar, por lo que apostar por una educación permanente y duradera a lo largo de su existencia, garantizara un mejor recorrido a nivel existencial y mayor facilidad para la adquisición y asimilación de los cambios producidos por nuestra evolución. 


\section{3 Ámbito de actuación}

Durante toda la vida, el hombre va pasando por varias etapas formativas hasta llegar a nivel deseado a nivel académico. Es verdad, que las primeras etapas educativas en la mayoría de países desarrollados pasan por ser obligatorias, formando parte de lo que llamamos educación formal, pero aparte de ésta, el hombre está influenciado a lo largo de su formación por otros dos tipos de educación, la no formal y la informal.

Estos tres tipos de educación tienen su papel específico y complementario en la formación del ser humano, siendo los tres necesarios para una formación plena del individuo. De esta manera podemos decir, que la educación formal equipará a la persona de los conocimientos y las calificaciones laborales que se solicitan en la sociedad, es competencia de la educación informal la de dotar al ser humano de un determinado número de aptitudes a nivel personal y social para su adaptación al entorno en el que vive, y por último, corresponde a la educación no formal, que la persona adquiera de una aptitud para vivir y de actitudes basadas en un sistema íntegro de valores.

\subsection{Educación Formal}

La educación formal, es aquella que se realiza en un sistema educativo institucionalizado, el aprendizaje ofrecido tiene un carácter estructurado y que concluye con una certificación regulado por organismos gubernamentales. El aprendizaje formal es intencional desde la perspectiva del alumno.

Abarca diferentes niveles de educación desde la Educación Básica, hasta los estudios universitarios. El tránsito del educando por esta estructura se realiza mediante un sistema de credenciales, grados, títulos y certificados que acreditan los progresos y le permiten el pasaje a un nivel superior.

\subsection{Educación no Formal}

La educación no formal comprende toda actividad educativa organizada y sistemática realizada fuera de la estructura de un sistema formal. El aprendizaje no es ofrecido por un centro de educación o formación y normalmente no conduce a una certificación, pero conserva el carácter estructurado de la educación regada, desarrollado, generalmente, en contextos fuera del ámbito de la escolaridad obligatoria, pero existiendo una intencionalidad educativa y una planificación de las experiencias de enseñanza- aprendizaje. El aprendizaje es intencional desde la perspectiva del alumno para impartir ciertos tipos de aprendizaje sea cual fuere su edad, y normalmente con este tipo de formación se adquiere una serie de aptitudes para vivir, basadas en un sistema íntegro de valores.

La educación no formal tiene la característica de estar dirigida a un gran abanico de destinatarios, está vinculada a la educación permanente y parte de las necesidades e inquietudes de los receptores para su desarrollo. Son los discentes los que 
optan de manera libre de estudiar este tipo de educación, a través de cursos de formación de adultos, seminarios, congresos, actividades institucionales, etc.

\subsection{Educación Informal}

A través de la formación informal cada persona adquiere y acumula conocimientos, capacidades, actitudes y comprensión a través de las experiencias diarias y del contacto con su medio. Se desarrolla en un ámbito extraescolar sin ningún tipo de planes de estudio ni acreditación directa. El personal que actúa como agente educador no es profesional, sino que puede ser de diversa índole, familiares, compañeros, mass media, y es a través de la interacción diaria con acciones de animación, difusión, estímulo, promoción como se van trasmitiendo una serie de contenidos, llegando a ser más eficaz que otros aprendizajes, porque aparece como una iniciativa del propio sujeto, en base de una motivación interna o interés.

A nivel general, los conocimientos que desde la educación informal recibe una persona a lo largo de toda su vida son muchísimos, siendo de gran importancia por su relevancia en el día a día.

\subsection{Formación de personas adultas}

La educación de adultos pertenece al ámbito de la formación no formal, cuya misión es de dotar a los adultos de las competencias necesarias para realizar una determinada acción, así como dotarles de conocimientos, actitudes y habilidades para el desarrollo de cualquier actividad que han optado por libertad o necesidad.

La formación permanente se le asocia mayormente con la educación de adultos, por considerarse a este colectivo, como un grupo que ha terminado su educación reglada y quiere seguir formándose a nivel no formal en competencias especificas, o incluso aquellas personas que no pudieron obtener la credencial de los estudios primarios, y por esta vía puedan acceder a alcanzar los estudios deseados.

Cuando hablamos de adultos, nos referimos a aquella persona con una determinada madurez personal, psicológica y social, que puede acometer actividades que legalmente son autorizadas. Al contrario que los estudiantes de niveles inferiores de la educación reglada, este colectivo posee una serie de competencias necesarias y susceptibles de cambio en mejora de adquirir las competencias perseguidas. Desde esta perspectiva, consideramos la educación de adultos como una formación que prepara a la persona para la anticipación y la innovación, un sistema de educación que da respuesta a sus necesidades, dentro de una sociedad de cambios constantes.

Tal y como apunta Escotet (1990), la educación es un camino sin fin y el aprendizaje es un viaje continuo donde no tiene sito el retorno, de tal manera, dependerá del sistema educativo proporcionar los instrumentos necesarios, así como las metodologías y especialistas, para adaptar las transformaciones sociales a cada uno de los distintos colectivos, como hemos mencionado anteriormente, preparando al adulto 
anticipándose a los futuros avances sociales (Escotet, 1990).

El hombre adulto se desenvuelve en una etapa cronológica en la que interactúan sus bases biológicas y el aprendizaje, todo mediado a través del ambiente social y cultural, por lo que se hace necesario, la formación de adultos partiendo de estas necesidades y explotando las competencias adquiridas a lo largo de su experiencia, y no partir de los déficits, como apuntan muchas teorías pedagógicas y psicológicas.

La educación de adultos, tiene como función la de dotar al adulto de una formación suficiente, que le dote de las herramientas necesarias para poder afrontar los objetivos fijados, por ello, una formación permanente en este colectivo, fomenta y desarrolla las competencias básicas, que no han podido adquirir en la enseñanza básica reglada, también desarrolla la formación en competencias más genéricas, adaptadas a los intereses y necesidades del adulto en su quehacer diario dentro del mundo laboral, y por supuesto con la adquisición de otras competencias a nivel instrumental, sistémica o técnicas, que ayudaran a su crecimiento personal y social, y a su participación más fructuosa en el mundo laboral, sin con ello, eliminar todo el conjunto de experiencias y competencias adquiridas a través de los años vividos.

Aunque la educación de adultos es una de las modalidades de formación que disponemos para desarrollar una educación a lo largo de la vida, es necesario también, hacer práctica lo que apunta Delors (1996) en relación a los cuatro pilares básicos de tiene que tener la educación y la formación en nuestros días, que son:

a) Aprender a vivir juntos, adquiriendo con ello valores como respecto, solidaridad empatía para conseguir una convivencia en paz.

b) Aprender a conocer, sobre todos los conocimientos básicos que conformen la base de nuestro aprendizaje a lo largo de la vida.

c) Aprender a hacer, en relación a la adquisición de las competencias necesarias para conservar y transformar nuestra sociedad en vías al bienestar.

d) Aprender a ser, fomentado el respeto hacia uno mismo y hacia los demás, siendo responsable, honesto y justo con lo que nos rodea, creando un mundo mejor. (Delors, 1996)

\section{METODOLOGÍA}

El propósito de la presente investigación es exponer la metodología conceptual y el esquema lógico conductor para el desarrollo de un proyecto social que contribuya al adulto mejor mejorar su bienestar social, laboral y personal a través de un conjunto de estrategias sustentadas en la formación educacional.

Para el diagnóstico de la situación problémica se emplearon como método teórico: el análisis documental, mediante el cual se consultaron fuentes especializadas provenientes de múltiples bases de datos como es el caso de la base de datos: Cambridge Scientific Abstract (Social). 
Otro de los métodos teóricos empleados para el análisis la propuesta del proyecto, fue el método deductivo para determinar la secuencia de actividades y la metodología para la implantación del proyecto.

\section{ANÁLISIS Y DISCUSIÓN}

\subsection{Proyecto de innovación educativa}

Una vez visto sobre qué marco teórico se mueve la educación a lo largo de la vida, en este apartado se hace un análisis sobre la futura implementación de un proyecto de investigación, sobre la formación en técnicas de estudios para preparar la prueba de acceso a la universidad para personas mayores de 25 años.

\subsubsection{Antecedentes}

El ser humano está en constante evolución, conforme va creciendo necesita recursos, aprendizajes, respuestas, información, etc., es por ello necesario, la instauración de un plan de formación continua que ayude al ser humano a seguir formándose una vez terminada las enseñanzas regladas.

Normalmente, los que quieren acceder a la universidad a través de la prueba de acceso a mayores de 25 años, se interesan por las materias que tienen que examinarse con el fin de prepararse el examen en cuestión, pero la mayoría carecen de un hábito de estudio diario dispone de una metodología de estudio antigua o deficiente, o simplemente, no poseen ningún tipo de método de estudio, sino que se enfrentan al aprendizaje mediante la universal técnica de la memorización.

Ante esta situación, es necesario tomar medidas y dotar a estas personas de una base a nivel de estudio, para que su adaptación a la universidad sea lo más optima posible, es por ello, que un curso en estrategias de aprendizaje, no solo le facilitar el proceso de enseñanza/aprendizaje para superar la prueba selectiva, sino que también será la base en la cual edifique su posterior estudio superior.

Antes de edificar un bonito edificio, este tiene que tener una buena base y estructura, para que luego no se venga abajo con la decoración exterior, de igual manera, el ser humano tiene que tener una buena base cognitiva en herramientas, métodos y técnicas de estudio, para que el aprendizaje en sí sea significativo, es importante enseñar a saber aprender.

Por lo general, el colectivo que pretende acceder a la universidad a través de la prueba de acceso de mayores de 25 años, vienen del ámbito laboral y de estar largos periodos sin formación permanente, por lo que el volver a empezar a retomar el estudio diario, se convierte en una ardua actividad diaria, que muchas veces lleva a la angustia, desesperación, estrés, desesperanza en conseguir los objetivos deseados. 
Pues desde esa base se inicia un proyecto de innovación en educación, cuya finalidad es la de preparar a los que quieren acceder a la universidad a través de la prueba de acceso de mayores de 25 años, a conseguir las metas propuestas, a dotarla de las herramientas necesarias para poder adquirir el aprendizaje de la manera más eficaz, eficiente y factible posible, sin olvidar, que el estudio es una tarea costosa al principio, pero luego lleva consigo bastante recompensas.

\subsection{Marco Teórico}

El proyecto de investigación sobre las estrategias de aprendizajes y técnicas de estudio para las personas que se preparan la prueba de acceso de mayores de 25 años a la universidad, estaría dentro del marco de la educación no formal, ya que se trata de un tipo de formación sistemática, de duración relativa y no esta circunscrita a la educación reglada.

Existen diferentes fuentes (Monereo, 1994; Pérez, 1995; Barriga \& Hernández, 2002) que afirman la necesidad de implementar métodos, estrategias y /o técnicas efectivas que sirvan a los estudiantes como herramientas básicas para la formación y el desarrollo de buenos hábitos de estudios, y observando el perfil de las personas a las que va dirigido este plan de formación, es importantísimo la adquisición de los mismos.

Lo que comentábamos de Escotet en líneas anteriores en relación a que la educación es un camino sin fin y cuyo aprendizaje no tiene carácter retroactivo, se magnifica en el colectivo de personas mayores de 25 años que acceden al plan formativo, porque se encuentra en una etapa cronológica en la que interactúan sus bases cognitivas y el aprendizaje, y todo envuelto en una ambiente social y cultural cambiante (Escote,1990).

Un factor también importante de este tipo de formación continua, es que tanto los tiempos y los ritmos de aprendizajes son flexibles, adaptados a las características de los destinatarios, así como al contexto donde se desarrolla la formación, ya que no tendrá el mismo ritmo de aprendizaje y tiempo disponible para la metacognición, las personas que se encuentren actualmente en situación activa laboral, como las que se encuentre en calidad de desempleado o venga de cursar enseñanzas, regladas o no, en otros ámbitos, con el consiguiente ritmo de estudio.

La estructura a desarrollar en este tipo de educación es variada y multidisciplinar, que dentro de la educación no formal, esta integradas todas las disciplinas, ciencias, letras, tecnologías, mundo laboral y social. Según la índole y tema a trabajar, los formadores podrán venir desde diferentes ámbitos, no estando tan limitado y especificado como en la educación reglada.

Al trabajar con un colectivo específico, como son las personas mayores de 25 años que quieren realizar la prueba de acceso a la universidad, los que implementaran el curso adaptaran los contenidos en materia de técnicas de estudio a las características del 
mismo, como puede ser la larga inactividad en el estudio, escaso o nada de habito en el estudio, herramientas y técnicas de estudio obsoletas, poca familiaridad con las nuevas tecnología en materia educativa, necesidad de reciclaje a nivel de estrategias de aprendizajes, etc.

Una de las finalidades que ofrece esta formación continua es capacitarlos y prepararlos en habilidades y destrezas básicas, para que puedan presentarse a la prueba de acceso con mayores garantías de superar la prueba. También fomentará el hábito al estudio y promoverá valores y actitudes hacia el perfeccionamiento de la persona humana y el desempeño de las aptitudes personales.

Según Olcese," los humanos somos criaturas de hábitos y estos son los que guían la mayoría de nuestros actos", por lo que es necesaria la instrucción y la adquisición de hábitos en el estudio para hacer el aprendizaje más efectivo. Todas las tareas que normalmente realizamos diariamente se convierten en hábitos, con más fundamentos, tenemos que preparar a estas personas para su acceso a la universidad con las herramientas necesarias para conseguir sus fines (Olcese, 2009).

Siguiendo con Luetich (2002), las técnicas de estudios son maneras de hacer operativa nuestra capacidad frente al estudio y el aprendizaje. Ayudan a la atención y a la concentración, distinguiendo lo que es principal de lo secundario, e implica a la escritura, conjuntamente con lo visual y auditivo, reduciendo la dispersión. Estas pautas son necesarias que la adquiera el alumno, para facilitar el proceso de aprendizaje, y debido a la ausencia, olvido, desuso del hábito de estudio, es necesaria una nueva reorientación en estas estrategias para que todo el conocimiento a adquirir sea más sencillo, coordinado y enriquecedor Luetich (Luetich, 2002.

Siguiendo a Mahillo "las técnicas de estudio son una herramienta útil para aprender y sacar mejores notas con menor esfuerzo", con las estrategias y técnicas a enseñar, no se pretenderá ofrecer a los alumnos de un recetario, o un modelo único de aprendizaje, sino que se le dotará de una serie de herramientas que puedan adaptarla a su propia (Mahillo, 1993, p.22) idiosincrasia y la utilicen de manera efectiva y optima para rendir lo más satisfactoriamente posible.

No todos los modos de hacer las cosas son iguales de eficaces, sino que existen unas maneras más beneficiosas y eficaces que otras, es importante que los alumnos descubran estos, de manera que adopten hábitos de estudios eficaces para no sucumbir en el arduo mundo del estudio con resultados poco efectivo.

\subsection{Camino de la Formación}

Siempre me ha resultado interesante el dicho de "no te acostaras sin saber algo nuevo", pues podríamos decir que este refrán es la piedra angular del proyecto de investigación. La vida está llena de conocimientos, información, innovaciones, nuevos procesos de interacción y comunicación, etc., por ello es necesario tener una buena base intelectual para que la adquisición y la adaptación de estos nuevos saberes, sea lo 
más fructífera y eficientemente posible.

Este proyecto persigue dar respuestas a cuestiones como ¿para qué estudiar? ¿Por qué tengo que estar siempre formándome? ¿Qué ventajas tiene la formación continua? ¿Cuándo terminaré de estudiar? ¿Qué tipo de formación es la más apropiada a mis intereses? ¿Cómo preparar la prueba de acceso de mayores de 25 años a la universidad? ¿Necesito seguir un plan de estudio para conseguir mis objetivos?

Es necesario informar a la sociedad de lo importante que es seguir formándote a lo largo de toda su vida, de las ventajas que tiene la formación continua en el desarrollo personal de la persona en todos los ámbitos, desde la formación ocupacional, buscando el perfeccionamiento en vías de prosperar laboralmente, hasta la formación en la empresa, con el fin de realizar el trabajo de manera más eficiente y eficaz. En la educación todavía no se ha tocado techo.

Desde que nacemos estamos en continuo proceso de aprendizaje, explorando, investigando, construyendo nuestra realidad, pero este proceso no se termina cuando has finalizado estudios superiores o has abandonado los estudios reglados, sino que el ser humano está en constante evolución, necesita seguir formándose como persona, como ser social, como ciudadano de la tierra. La educación es para toda la vida, es parte intrínseca de nosotros, y es oportuno informar de todo lo positivo que podemos integrar a nuestra vida por medio de la educación, educar es promover el desarrollo de una persona, formar, instruir, guiar en el proceso de la vida.

Una vez que hemos cortado nuestros estudios por realizar otras actividades, (trabajo, ocio, asuntos personales, etc.) nos encontramos con el inconveniente, que cuando volvemos otra vez a retomarlo, necesitamos reiniciar, ya que los hábitos de estudios que teníamos anteriormente se han olvidado o se han quedado obsoletos. Muchos de los problemas con los que se encuentran aquellas personas que deciden volver a estudiar y proseguir con la enseñanza superior, es que no disponen de una serie de competencias educativas para superar la primera prueba a esos estudios, como es la prueba de acceso a mayores de 25 años.

Un alto porcentaje de personas que se presentan a esta prueba, lo hacen por intereses varios, como prosperar laboralmente, autorrealización personal, o por ser una de las únicas vías posibles de seguir creciendo ante la realidad social.

El trabajar de nuevo con estas personas cuestiones como el lugar de estudio, mobiliario y condiciones físicas optimas para el buen rendimiento, métodos de estudios, la memoria, preparación antes y durante los exámenes, etc., son pautas muy enriquecedoras que no solo los prepara para presentarse a la prueba de acceso, sino que también formaran la base de estudio para su posterior carrera universitaria.

Necesitamos seguir estudiando toda nuestra vida, y el poseer un buen habito de estudio como técnicas y metodología apropiadas, con la finalidad de que nos faciliten 
nuestra incorporación y desarrollo en los estudios superiores y en todas las ramas de nuestra sociedad, ya que siempre se necesita actualizarse, adaptarse a las nuevas tecnologías, y teniendo una estructura cognitiva entrenada, preparada, nos facilitara seguir trabajando para nuestro bienestar personal y social.

Tal y como hemos mencionado en líneas anteriores, nos encontramos en una sociedad de cambios y transformaciones, donde el ritmo de las innovaciones y los avances tecnológicos, sociales, políticos, etc., cada vez son más constantes y rápidos, y ante esta situación, es necesario una educación permanente que ayude a solventar estas necesidades y apoyar al hombre en su adaptabilidad, sin con ello, tener que desprenderse de su hábitos diarios.

El colectivo al que va dirigido el proyecto de investigación, son personas adultas mayores de 25 años, que por diversos motivos, quieren acceder a estudios universitarios, y aunque se encuentran cursando un curso de preparación para la prueba de acceso, requieren técnicas y estrategias de aprendizaje, que les ayude a la hora de asimilar conocimientos y habilidades para dicha prueba, y además configuren una buena base seguir con sus estudios superiores.

Las personas adultas, tienen unas características psicosociales especificas, su proceso de aprendizaje es diferente al que efectúan los alumnos en etapas escolares, por lo que es conveniente adaptarse a sus necesidades y competencias adquiridas a través de sus experiencias laborales, personales y sociales, y partir de ellas, poder configurar un nuevo marco de enseñanza/aprendizaje.

La formación permanente que se le ofrece con un curso de formación, es la de dotarle con técnicas, herramientas, instrumentos de adquisición de procesos cognitivos, habilidades, procesamientos de información, etc., que le ayuden a la asimilación de los conocimientos y el consiguiente aprendizaje significativo, que le aportara la superación de la prueba de acceso a la universidad. Es importante, resaltar, que este tipo de curso de formación en técnicas de estudios, está centrado en las características de este colectivo, parte de las competencias, la experiencia, el contexto social y laboral que se encuentra los alumnos, así como adaptado a los ritmos y tiempos de los mismos, por ello, la modalidad de formación es a distancia.

Este colectivo por diferentes factores, han interrumpido su educación reglada, y tras unos años de inactividad académica, se sumergen de nuevo en el mundo de la educación, encontrándose con la dificultad de que carecen de estrategias de aprendizajes apropiadas para los nuevos conocimientos, o que sus técnicas de estudios están obsoletas, o incluso, carecen totalmente de una metodología de estudio apropiada para los fines perseguidos.

El curso de formación se encuentra situado dentro de la educación no formal, cuenta con una estructura organizativa y sistemática y el aprendizaje es demandado por el propio alumno, ocupando con ello la motivación, un referente y punto de apoyo de relevancia, por los fines e intereses que persiguen. El curso no otorga finalmente 
ningún tipo de credencial, pero es de un gran valor el resultado que en los alumnos aporta al cursarlo y adquirir los conocimientos del mismo, gracias a los profesionales de la educación que han confeccionado y llevado a la práctica dicho plan formativo.

Una de las competencias que se va trabajar con los alumnos a través del plan de formación, además de asentar y afianzar las competencias básicas, que por lo general son desarrolladas en las etapas de escolaridad obligatoria, serán las competencias genéricas en hábitos y técnicas de estudios, que se considera necesario para que los alumnos adopten todas las habilidades, conocimientos, actitudes, etc., para desarrollar a posteriori las actividades perseguidas, que no son otras, que estudiar.

Como indica Albert "entendemos por formación continua el conjunto de acciones formativas orientadas tanto a la mejora de las competencias y cualificaciones profesionales como a la recualificación y actualización de los trabajadores ocupados" (Albert 2009, p.206), en este sentido considero la preparación de la prueba de acceso a la universidad como un tipo de actividad encaminada a la actualización y mejora laboral, ya que lo que se pretende es acceder a la universidad para poder mejorar la situación laboral, y en este ende, el curso de formación es la mejora formativa, en el sentido de formación continua, que le ayudara a superar la prueba de acceso, pero también le acompañara a lo largo de toda su vida labora pudiendo aplicar estos conocimientos en cualquier rama profesional, a la cual definitivamente vaya a desempeñar su labor.

Finalizando, la educación a lo largo de vida significa una educación permanente que comienza en las primeras etapas de nuestra vida y nos acompaña hasta el final de nuestros días. La formación recibida tiene que estar presente a lo largo de nuestra carrera profesional, y en ese sentido, el apostar por tener una base solida para desarrollar un aprendizaje significativo, disponer de herramientas de procesamiento de los conocimientos, actitudes y habilidades que se actualizan diariamente, y el proyecto formativo que se oferta cumple con estos requisitos e incluso aporta unos cimientos para poder ir adaptándonos a los cambios de nuestra sociedad.

\subsection{Finalidad del proyecto}

El objetivo del proyecto de innovación educativa, es la preparación en materia de técnicas de estudio de personas mayores de 25 años para la prueba de acceso a la universidad.

Se debe de planificar un curso en el cual, se aborden técnicas de estudios y estrategias de aprendizajes para facilitar, la adquisición y procesamiento de la información entrante mediante el estudio, para su aplicación exitosa tanto para la prueba de acceso como para la consiguiente carrera formativa universitaria posterior.

Los objetivos a que se tienen que cumplir, para que los alumnos puedan conseguir las competencias a nivel de capacidades cognitivas operativas, serian las siguientes: 
a) Adquirir técnicas y estrategias de aprendizajes para estudiar.

b) Elaborar una planificación del trabajo.

c) Conocer las condiciones ambientales, personales y psicosociales adecuadas para el estudio.

d)Saber utilizar de métodos de estudios adaptados a la idiosincrasia del alumno

e) Adquirir competencias en materia de lectura, toma de apuntes, elaboración de resúmenes y mapas conceptuales

f) Conocer las pautas a seguir antes y durante los exámenes

La finalidad del proyecto innovador educativo es el desarrollo de un curso formativo, dentro del ámbito de la educación no formal, donde los discente se le habiliten de herramientas, técnicas, de una metodología que le facilite la adquisición de conocimientos, proporcionales pautas de aprendizaje para hacer más eficiente el estudio, formar en ellos hábitos de estudios que puedan utilizar para prepararse la prueba de acceso a la universidad, y luego en la universidad.

\subsection{Metodología.}

Hablamos de metodología cuantitativa cuando utilizamos técnicas y recursos en los que se trabaja con datos cuantitativos, mientras la metodología cualitativa evita esta cuantificación. La investigación cualitativa se mueve hacia la búsqueda de registros narrativos de los fenómenos que son estudiados mediante técnicas como la observación participante y las entrevistas no estructuradas. Las investigaciones cuantitativas buscan el dato, lo empírico, la comprobación racional de lo estudiado.

Una de las diferencias de estas investigaciones es que la cualitativa trata de identificar la naturaleza profunda de las realidades, su sistema de relaciones, su estructura dinámica y la investigación cuantitativa trata de determinar el porqué ocurren las cosas de una manera determinada a través de la objetivación y generalización de los resultados.

La metodología a desarrollar durante el proyecto educativo será de índole cualitativa, ya que estamos trabajando con personas, pero obedecerá al contexto y a las características de los destinatarios. Es importante comenzar desde un análisis de las necesidades de los alumnos que acceden al curso y adaptar nuestro proceso de enseñanza/aprendizaje a sus características, al entorno donde se desenvuelve, a sus ritmos de aprendizajes, a su nivel cognitivo de partida.

La metodología cualitativa se centra el a investigación que produce datos descriptivos, como las propias palabras de las personas, habladas o escritas, como la observación de la conducta. Las características de esta metodología que nos ayudan en el proceso de enseñanza/aprendizaje a la hora de implementar el curso, por su cualidad inductiva, holística, capacidad de interacción, comprensión y flexibilidad a la hora de la interpretación. Como afirma Blumer (Blumer ,1996), los métodos cualitativos nos van a permitir estar próximos al mundo empírico (Blumer ,1996).

Se utilizara una metodología participativa, donde la comunicación entre formador y 
formados sea bidireccional, apoyados por clases magistrales y supuestos prácticos y actividades para afianzar los conocimientos propuestos.

Factores como la motivación, el esfuerzo, la voluntad, etc., serán palabras claves con el propósito de fomentar la autorrealización, la crítica constructiva personal y la consecución de los logros establecidos.

Principios como individualización, socialización, actividad, creatividad, etc., también tendrán cabida en el proceso de enseñanza/aprendizaje, ya que se trata de que los alumnos adquieran de forma autónoma e independiente del conjunto de saberes que le proporcione un mejor rendimiento, pero sin olvidar que el contexto de aprendizaje se da de forma colectiva, donde la socialización, la participación y el trabajo en grupo, también proporciona un enriquecimiento educativo a nivel personal.

La metodología que sustentara el proyecto de investigación estará basada en la colaboración entre alumnos y profesores, procurando las responsabilidades individuales y sociales, donde se sustituirá la competitividad por cooperación, la coeducación, en la que cada alumno ejercerá sobre el otro una influencia saludable, y donde se prepare al individuo para ser no solamente ciudadano capaz de cumplir con sus deberes sino también ser humano consciente de su dignidad de persona.

También se fomentara el proceso de enseñanza/aprendizaje mediante la utilización de técnicas de dinámicas de grupos como mesa redonda, Philips 6.6, técnicas de discusión y debate, role-playing, estudios de casos, foro, torbellinos de ideas, análisis, etc.

Dentro de ese curso de formación también tendrá cabida la metodología cuantitativa con el fin de contar con datos que nos faciliten y nos den información sobre la consecución de las metas perseguidas, y la utilización de técnicas cuantitativas como cuestionarios, test, y otro tipo de herramienta que nos permitan cuantificar la información obtenida, serán de importancia a la hora de la evaluación.

\subsection{Esquema conceptual}

Las palabras claves dentro de la educación a lo largo de la vida, serian la educación no formal, como referente dentro de los ámbitos de la educación, la formación de las personas adultas, como colectivo a cual va dirigida el plan de formación, la formación permanente, como la educación a la cual tiene que estar ligado la formación de los adultos y por supuesto la persona adulta. La educación no formal es el mejor ámbito de actuación para desarrollar en el colectivo de las personas adultas, hábitos de estudios, estrategias cognitivas de aprendizajes y patrones educativos que puedan usar y adaptarse a sus propias necesidades y demandas personales. Además lo que se persigue con el curso, es que la formación recibida sea continuada y aplicable durante su formación posterior, conformando con ello una formación continua en base a la adquisición de nuevas experiencias y conocimientos.

A continuación se detalla en forma de esquema conceptual todo lo tratado a lo largo 
del trabajo.

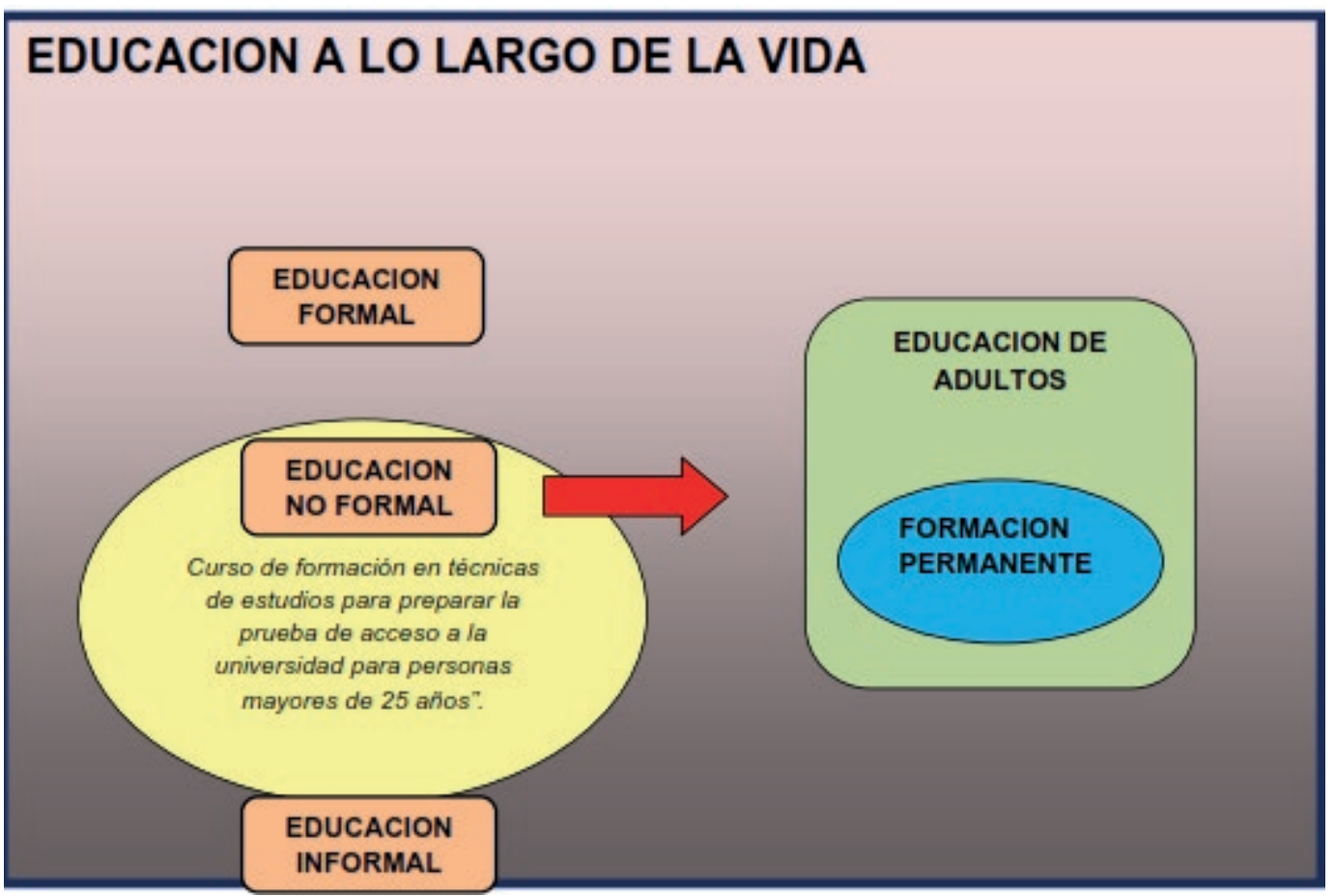

Figura 1. Esquema conceptual del proyecto.

El análisis de este mapa conceptual engloba que se entiende como educación a lo largo de la vida. Observamos como durante toda la vida, los tres ámbitos de educación nos acompaña en todo nuestro aprendizaje, pero es necesario hacer hincapié, en el hecho, que el plan de formación se encuentra situado dentro del ámbito no formal de la educación, pero también toca un poco del ámbito informal, ya que en el proceso de enseñanza/aprendizaje, las experiencias vividas, la interacción con los compañeros del cursos, así como las competencias adquiridas anteriormente, también juega un papel a la hora de la adquisición de las nuevas competencias.

También observamos como la educación no formal conlleva la educación de adultos, vista de manera de una formación permanente, que no acaba cuando se han conseguido las metas propuestas, sino que se va actualizando y la vamos sumando en nuestro día a día, ya que como comentábamos en líneas anteriores, esta formación recibida, es aprovechada en el futuro, por ejemplo, cuando se esté cursando los estudios universitarios.

\section{CONCLUSIONES}

A lo largo de todo el trabajo, hemos desarrollado una línea de interpretación en lo referente al significado de la educación a lo largo de la vida en las personas adultas, y tal como está enfocado el proyecto, especialmente al colectivo de adultos que se preparan la prueba de acceso a la universidad.

Uno no termina de formarse hasta que muere, por lo que la actualización siempre nos acompañara si queremos movernos conforme avanza nuestro mundo. Para ello, de 
todos los ámbitos educativos que nos encontramos, la educación no formal es el vehículo apropiado para que nos lleve hacia el destino de la formación permanente, teniendo en cuenta nuestras características como adultos, experiencias, competencias y habilidades ya asentadas en nuestro propio ser, es por esto que la educación debe enfocarse hacia la pautas de cambio, modificación o adaptación a los nuevos inputs que necesitamos tener, para conseguir las nuevas metas propuestas.

Dentro de la educación a lo largo de la vida, no podemos olvidarnos de la formación ocupacional y la formación en la empresa y organizaciones, ya que aunque nos hemos centrado en la formación continua, tenemos claros que toda educación persigue unos objetivos concretos, la formación para el desempeño de una cualificación profesional, donde la formación en la empresa y en la organizaciones juegan un papel importante en el tema de actualización y reciclaje de trabajadores en activos, la formación ocupacional que prepara a colectivos específicos de cara al mundo laboral, o simplemente la formación como medio de culturalizarse y obtener un bienestar cultural personal.

Sumando todas las ideas se puede llegar a la conclusión, que la educación es el motor de nuestra sociedad, es la llave del conocimiento, y hasta se puede decir que es el kit de supervivencia en un mundo que avanza sin mirar atrás. La formación continua, permanente, la educación a lo largo de la vida, es un proyecto necesario e imprescindible para el bienestar personal y social de la persona.

\section{BIBLIOGRAFÍA}

Albert, M.J. (2007). La investigación Educativa. Claves teóricas. Madrid. McGraw- Hill.

Bernardo, J. (2004). Estrategias de aprendizaje. Para aprender más y mejor. Madrid: Rialp.

Bloom, B.s.hasting, J.T. Y MadauS, G. (1975). Evaluación del aprendizaje. Buenos Aires: Praxis.

Cubero, R. (1993). Como trabajar con las ideas de los alumnos. Sevilla: Diada

Delors, J. (1996). La educación encierra un tesoro. Informe a la UNESCO de la Comisión Internacional sobre la educación para le siglo XXI. Madrid: Santillana EdicionesUNESCO.

Delval, J. (1990). Los fines de la educación. Madrid: Siglo XXI.

Gagne, R. Y Briggs, L. (1974). Planificación de la enseñanza. México: Trillas

Escotet, M.A. (1990). Visión de la Universidad del S. XXI, dialéctica de la misión universitaria en una era de cambio. Revista Española de Pedagogía, 86, 211-228.

Ferrandez, A. (1990). Realidad y perspectivas de la educación de personas adultas.Madrid: Fondo Formación. 
Ferrant Jimenes, E. \& Wilfredo Salas, M. (2007). Adquisición y formación de hábitos de estudio para un aprendizaje eficiente. México: Universidad Veracruzana.

Jimenez Ortega, J. y Alonso Obispo, J. (1997). Aprendiendo a estudiar. Madrid: Akal.

Longworth, N. (2003). El aprendizaje a lo largo de la vida. Ciudades centradas para un siglo orientado hacia el aprendizaje. Barcelona: Paidós

Mahillo, J. (1993). Sabes estudiar? Madrid: Espasa Calpe.

Murga, M.A. (Coord.) (2009). Escenarios de Innovación e Investigación educativa. Madrid: Universitas.

Olcese Salvatecci, A. (2009). Como estudiar con éxito: técnicas y hábitos para aprender mejor. Barcelona: Alfaomega

VV.AA. (1996). Técnicas de estudio. Madrid: Radio Ecca.

\section{Francisco Manuel Moreno Lucas}

Licenciado en Pedagogía en la Facultad de Educación de la Universidad de Murcia. Está en posesión del Máster Universitario en Innovación e Investigación en Educación impartido por la UNED, además del máster en Dirección y Gestión de Recursos Humanos impartido por CEF y el curso superior en Gestión Laboral y Seguridad Social por IMF. Actualmente se encuentra trabajando como tutor personal desde 2010 en la Universidad Católica San Antonio de Murcia, y colabora como profesor en la asignatura de Sociología de la Empresa que se cursa en la Licenciatura de Administración y Dirección de Empresa de esta universidad. 